\title{
Einleitung: Entwicklung und Perspektiven der Internationalen Politischen Ökonomie (IPÖ) nach der Weltfinanzkrise
}

\author{
Hans-Jürgen Bieling • Tobias Haas • Julia Lux
}

\section{Die Genese einer neuen Interdisziplin}

Bei der Internationalen Politischen Ökonomie (IPÖ) handelt es sich sowohl um eine sehr alte als auch recht junge Disziplin im Schnittfeld von politik- und wirtschaftswissenschaftlichen Themen und Fragestellungen. Sie ist sehr alt, da sich bereits die Klassiker der Politischen Ökonomie - Adam Smith, David Ricardo, Friedrich List, Karl Marx, Karl Polanyi etc. - mit grenzüberschreitenden, mithin trans- und internationalen Dynamiken befasst hatten. Sie ist aber insofern recht jung, als die IPÖ erst seit den 1970er Jahren als ein klar identifizierbarer Forschungsbereich in der akademischen Welt institutionalisiert wurde. Dieser Institutionalisierungsprozess erfolgte zunächst im anglo-amerikanischen Raum und reflektierte insbesondere die sich mehrenden internationalen politökonomischen Umbruchtendenzen, etwa den raschen Aufholprozess Westeuropas und Japans gegenüber den USA, die wachsende Bedeutung transnationaler Konzerne, den Zusammenbruch des Bretton-Woods-Systems oder auch die weltwirtschaftlichen Krisenprozesse der 1970er Jahre. Diese und andere Entwicklungen veranlassten zunächst einige - Benjamin Cohen (2008) identifiziert sieben „glorreiche Gründungsfiguren“: Robert Keohane, Robert Gilpin, Susan Strange, Charles Kindleberger, Robert Cox, Steven Krasner und Peter Katzenstein - und schließlich mehr und mehr WissenschaftlerInnen, sich der Analyse politökonomischer Phänomene zuzuwenden und die IPÖ als aufkeimende Interdisziplin durch Publikationen, Forschungsprojekte, akademische Sektionen und neue Studiengänge im Wissenschaftssystem zu verankern.

\footnotetext{
Online publiziert: 13.11 .2013

(C) Springer Fachmedien Wiesbaden 2013

Prof. Dr. H.-J. Bieling $(\bowtie) \cdot$ T. Haas · J. Lux

Institut für Politikwissenschaft, Universität Tübingen,

Melanchthonstr. 36, 72072 Tübingen, Deutschland

E-Mail: hans-juergen.bieling@uni-tuebingen.de

T. Haas

E-Mail: tobias.haas@uni-tuebingen.de

J. Lux

E-Mail: julia.lux@uni-tuebingen.de
} 
Im Vergleich zur anglo-amerikanischen Diskussion erfolgte die Institutionalisierung der IPÖ in Kontinentaleuropa, so auch in Deutschland, mit einer gewissen Zeitverzögerung (Bieling 2011; Schirm 2013). Um nicht missverstanden zu werden: An Debatten und analytischen Beiträgen bestand auch hier in den 1970er Jahren kein Mangel. Als eine eigenständige Interdisziplin konnte sich die (Internationale) Politische Ökonomie aber nicht etablieren, da der Begriff aufgrund der Assoziation mit neo-marxistischen Analyseperspektiven vielfach Abwehrreaktionen hervorrief. Diese Abwehrhaltung ließ in dem Maße nach, wie auch hierzulande die mitunter sehr inspirierenden internationalen Diskussionen zur Kenntnis genommen wurden und die forcierte Globalisierungsdynamik seit den 1980er Jahren die sozialwissenschaftliche Forschung mit politökonomischen Problemen und Krisenprozessen konfrontierte. Gestützt auf einige Professuren, Forschungsund Arbeitszusammenhänge sowie eine wachsende Zahl an Publikationen, Tagungen und einzelnen Studiengängen schreitet die Etablierung der IPÖ auch in Deutschland seit einiger Zeit voran.

Thematisch ist die IPÖ sehr breit aufgestellt. Sie befasst sich im Prinzip mit allen Phänomenen der Globalisierung, von den Handelsbeziehungen, der Produktionsorganisation und den Finanzmärkten über die Arbeitsbeziehungen und Wohlfahrtsstaaten bis hin zu internationalen Übereinkommen und Regimen, einschließlich der veränderten Formen der staatlich-privaten Kooperation und der Herausbildung transnationaler politischer Autorität und Kontrolle. Auch räumliche, mithin geographische oder sozialkulturelle Aspekte spielen vielfach eine Rolle. Dies lässt erkennen, dass sich die IPÖ als Interdisziplin keineswegs nur an der Schnittstelle von Ökonomie und Politikwissenschaft bewegt, sondern auch - zumindest zum Teil - Fragestellungen und Impulse benachbarter Fächer wie etwa der Soziologie, der Wirtschafts- und Humangeographie oder auch der Geschichtswissenschaft aufgreift. Die interdisziplinären Anregungen mögen eine strikte Definition oder sogar Kanonisierung des Forschungsfeldes erschweren. Vor allem aber stimulieren sie lebhafte Diskussionen und neue Forschungspraktiken. Hierbei werden auch die alten Pfade, die die IPÖ-Debatte über einen langen Zeitraum geprägt hatten, d. h. die Untergliederung des Forschungsfeldes in eine merkantilistisch-realistische, eine liberal-institutionalistische und eine historisch-materialistische IPÖ (Gilpin 1987; Bierstecker 1993), vielfach verlassen. In diesem Prozess sind freilich auch neue Trennlinien und Abgrenzungen entstanden. So wurde in den vergangenen Jahren wiederholt darüber debattiert, ob, inwiefern und warum das Forschungsfeld durch eine transatlantische Kluft geprägt ist (Cohen 2007; Phillips und Weaver 2011). Auf der einen Seite ist die amerikanische IPÖ tendenziell durch ein akteurszentriertes Paradigma gekennzeichnet, das mit Blick auf die Trennung und Interaktion von Staaten und Märkten insbesondere die Interessen und zum Teil auch Diskurse der beteiligten AkteurInnen beleuchtet. Deren Relevanz wird dabei mithilfe spezifischer Modelle und Hypothesen empirisch erforscht und belegt. Diesem eher rationalistisch-positivistischen Zugriff steht auf der anderen Seite eine von Großbritannien ausgehende post-positivistische IPÖ gegenüber, die sehr viel stärker auch (trans-)nationale gesellschaftliche Strukturen und diesen eingeschriebene, d. h. durch Klassen, Gender und Ethnien konstituierte Machtbeziehungen und Diskurse in die Analysen mit einbezieht.

Diese Gegenüberstellung ist sicherlich - die kontroversen Reaktionen vieler prominenter WissenschaftlerInnen haben dies deutlich gemacht - stilisiert und etwas überzo- 
gen. Sie verdeutlicht zugleich aber auch, dass in der IPÖ sehr heterogene, nicht selten stark divergierende Analysen und Interpretationsangebote konkurrieren. Das vorliegende ZfAS-Sonderheft verfolgt in diesem Zusammenhang vor allem zwei Ziele. Das erste Ziel besteht darin, im Sinne eines state of the art ohne Anspruch auf Vollständigkeit ${ }^{1}$ einen breiten Überblick über die teils konkurrierenden, teils komplementären Perspektiven der deutschsprachigen IPÖ-Debatte zu geben. Das zweite, hiermit verknüpfte Ziel, macht darauf aufmerksam, dass ein solches Unterfangen derzeit nicht ganz leicht zu bewerkstelligen ist. Schließlich befinden wir uns in einer durch die Weltfinanzkrise von 2008/09 ausgelösten Krisen- und Transformationsphase, deren weiterer Verlauf in vielfacher Hinsicht ungewiss ist. Unter Berücksichtigung der offenkundigen Unsicherheiten geht es demzufolge darum, in einer Art Zwischenbilanz die Implikationen der Krisenprozesse für die Forschung und die IPÖ als Interdisziplin wie auch für den Forschungsgegenstand selbst, also die internationale politische Ökonomie (ipÖ), auszuleuchten.

\section{Die Weltfinanzkrise und die Folgen}

Die in diesem Heft versammelten Beiträge haben zwar jeweils einen spezifischen Fokus, wenden sich darüber hinaus aber allesamt den anhaltenden Krisenprozessen zu, über deren Ursachen und Charakter keineswegs Einigkeit besteht. In gewisser Weise ist dieser Sachverhalt in der Krise selbst angelegt. Denn per definitionem sind Krisen Phasen einer tiefen Erschütterung und Verunsicherung, also Zeiträume der Entscheidung oder potenziellen Wende, in der auch die tradierten Interpretationsmuster in Frage gestellt, zumindest aber überprüft werden. Im Unterschied zu den HistorikerInnen, die den Charakter und Verlauf einer Krise von ihrem Ende her bestimmen können, bewegen sich die übrigen Sozialwissenschaften, so auch die IPÖ, in der Jetztzeit. Dies hat den Nachteil, dass die unterbreiteten Krisendiagnosen unter den Bedingungen erhöhter Unsicherheit erfolgen und sich gegenüber konkurrierenden Analysen und Interpretationsangeboten behaupten müssen. Dies verdeutlicht: Krisen sind nicht einfach gegeben. Als Referenzpunkt der gesellschaftlichen Kommunikation und als Medium der Bearbeitung sozialer Prozesse, so bereits Reinhart Koselleck (1973, S. 105-146), sind sie vielmehr - nicht nur, aber auch - sozial konstruiert, ohne dass bereits klar ist, ob und in welche Richtung die diskursiven Konstruktionen letztlich wirken.

Ungeachtet der angesprochenen Unsicherheiten, analytischen Schwierigkeiten und kontroversen Interpretationen sind die politökonomischen Diskussionen durch eine übergreifende Grundauffassung gekennzeichnet. So wird allgemein davon ausgegangen, dass die Weltfinanzkrise der Jahre 2008/09 und die an sie anschließenden Krisendynamiken kein gewöhnliches, d. h. rasch vorbeiziehendes Phänomen darstellen. Es handelt sich nicht einfach nur um ein reinigendes Gewitter, nach dem business as usual betrieben werden kann, sondern vielmehr um einen Einschnitt, in dem die gesellschaftlichen Akteu-

1 Der Band beinhaltet keine Artikel, die sich schwerpunktmäßig mit Geschlechterverhältnissen, der Rolle Afrikas und den Nord-Süd-Beziehungen im Allgemeinen beschäftigen. Auch ist ein gewisser Eurozentrismus bei der Repräsentation der deutschsprachigen Debatte nicht zu vermeiden. 
rInnen und politischen EntscheidungsträgerInnen nicht umhin kommen, neue Weichen zu stellen und unbetretene Pfade zu beschreiten.

Gerafft stellt sich der Krisenverlauf wie folgt dar (Bieling 2009): Zunächst hatte die Fed, die US-amerikanische Zentralbank, nach dem Platzen der Dotcom-Blase zu Beginn des Jahrtausends das Wachstum durch niedrige Zinsen befeuert. In Verbindung mit der Deregulierung der Hypothekenkredite trug diese Politik des billigen Geldes mit dazu bei, dass sich eine Immobilienblase bildete. Als die ersten Kredite nicht mehr bedient werden konnten, folgte eine Kettenreaktion: Die Banken liehen sich kaum noch Geld, Immobiliengesellschaften mussten Insolvenz anmelden, Banken gerieten in Schieflage, die Investmentbank Lehman Brothers ging im September 2008 gar in Konkurs. Die Immobilienkrise weitete sich schnell zu einer Banken-, Finanz- und schließlich einer Wirtschaftskrise aus. Bereits im Jahr 2008 sank das BIP in den USA um 0,4\%, im Jahr darauf gar um $3,5 \%$. Im selben Jahr schrumpfte die Weltwirtschaft um 2,2\%, was verdeutlicht, dass die Krise globale Ausmaße erreicht hatte.

Die Übertragung der Krise auf andere Weltregionen verlief vornehmlich über zwei Kanäle: über die Kapitalmärkte und die Handelsbeziehungen (Bellofiore et al. 2010; Becker und Jäger 2012). Was die Kapitalmärkte betrifft, so waren vor dem Ausbruch der Krise die Forderungen aus Immobilienkrediten verbrieft und international gehandelt worden. Zahlreiche ausländische Banken, bis hin zu deutschen Landesbanken, besaßen solche Forderungen, die sie dann in erheblichem Maße abschreiben mussten. Neben den Kapitalmärkten sorgten auch die Handelsbeziehungen dafür, dass die Krise auf andere Weltregionen „überschwappte“. Im Zuge der Krise drosselten die USA ihre Importe zunächst deutlich, was sich insbesondere in exportorientierten, stark auf die USA ausgerichteten Volkswirtschaften niederschlug. In der Europäischen Union betrug der Rückgang des BIP im Jahr 2009 4,3\%, in Deutschland 5,1 \%. Diese Zahlen deuten an, dass die Krise im atlantischen Raum stärker durchschlug als in anderen Weltregionen.

Aufgrund des globalen Charakters der Krise wurden vor allem im Rahmen der G20Gipfeltreffen die Maßnahmen zur Bearbeitung der Krise koordiniert. Dabei lassen sich zwei Phasen der Krisenbearbeitung unterscheiden. In einer ersten Phase versuchten die Regierungen durch die Sozialisierung der Verluste der Banken den Finanzsektor zu stabilisieren und durch Konjunkturpakete Impulse für ein Anspringen des Konjunkturmotors zu setzen. Allerdings hat die massive Ausweitung der öffentlichen Verschuldung zumindest in den USA und besonders in der EU mit dazu beigetragen, dass diese erste Phase der Krisenbearbeitung von einer zweiten abgelöst wurde, die auf eine Konsolidierung der öffentlichen Haushalte mittels austeritätspolitischer Maßnahmen abzielt. Besonders in den Staaten der europäischen Peripherie, die aufgrund deutlich negativer Leistungsbilanzen ihre Staatsdefizite mittels eines „Risikoaufschlags“ refinanzieren mussten, wurden und werden strikte Austeritätsmaßnahmen durchgesetzt. Im Unterschied hierzu wurde die Krise in den aufstrebenden BRIC-Staaten relativ rasch überwunden und keine austeritätspolitische Konsolidierung eingeleitet.

Die Folgen der Weltfinanzkrise sind also räumlich höchst ungleich verteilt und unterscheiden sich erheblich von vorangegangenen Finanzkrisen. Die Finanzkrisen der vergangenen Jahrzehnte hatten alle ihr Epizentrum in der „Peripherie“ und konnten lokal begrenzt werden. Die aktuelle Weltfinanzkrise entwickelte sich hingegen vom Zentrum 
(den USA) aus und betrifft neben den USA besonders die EU. So kommt es, dass der IWF, der zeitweise Gefahr lief, in der Bedeutungslosigkeit zu verschwinden, ein Revival erlebt und als Teil der Troika (EU Kommission, EZB, IWF) die Länder der europäischen Peripherie mit Krediten versorgt und zugleich austeritätspolitisch diszipliniert.

Doch nicht nur in räumlicher, auch in politikfeldspezifischer Hinsicht divergieren die Auswirkungen der Krise erheblich. Während in manchen Politikfeldern die Krise nur geringe Auswirkungen zu haben scheint, sind in anderen Politikfeldern neue Dynamiken zu beobachten. Eine wesentliche Kontextbedingung der Krisendynamik besteht dabei in der Regulation der Finanzmärkte. Nach dem Zusammenbruch des Bretton-Woods-Systems, dessen zentrale Eckpfeiler das System fester Wechselkurse mit dem US-Dollar als Leitwährung und dessen Golddeckung bildeten, wurden in der Folgezeit die Finanzmärkte zunehmend dereguliert und die Spekulationen ausgeweitet, was in der Krise vermehrt kritisch betrachtet und diskutiert worden ist. Die Aufmerksamkeit richtete sich aber nicht nur auf den Bereich der Finanzmarktregulation, sondern auch auf die Implikationen der Krise für andere Politikfelder, wenngleich diese sehr unterschiedlich betroffen waren und sind.

Innerhalb der IPÖ divergieren die Bewertungen der Krise; dies hängt auch mit der Heterogenität der Disziplin und den verschiedenen Analyseperspektiven zusammen. Je nachdem ob man eher einem positivistisch akteurszentrierten Ansatz oder postpositivistischen Ansätzen zuneigt und welcher Gegenstandsbereich der IPÖ analysiert wird, kann die Einschätzung, welche Rolle die Weltfinanzkrise für die Entwicklung der Weltwirtschaft und die IPÖ spielt, höchst unterschiedlich ausfallen. Einigkeit besteht jedoch darin, dass die Weltfinanzkrise ein ganz zentrales Ereignis für die IPÖ-Forschung darstellt und zugleich die Möglichkeit eröffnet, die eigenen Forschungsansätze kritisch zu reflektieren, Queen Elizabeths im Jahr 2008 an der London School of Economics aufgeworfener Frage folgend: „Why did nobody see it coming?“ Insofern kann sich die Weltfinanzkrise auch als Auslöser einer produktiven Theoriedebatte entpuppen. Vielleicht stellt die Weltfinanzkrise für die IPÖ sogar eine Zäsur dar, die mit dem Ende des Ost-West-Konflikts und den entsprechenden Folgen für die IB-Forschung vergleichbar ist.

Gleichwohl ist es noch zu früh, die Auswirkungen der Krise auf die Forschung und auch auf den Forschungsgegenstand endgültig festzustellen, denn gegenwärtig herrscht innerhalb der Disziplin der IPÖ keine Einigkeit darüber, ob wir uns in oder nach der Weltfinanzkrise befinden. Nichtsdestotrotz lassen sich zumindest zwei generelle Tendenzen für die IPÖ beobachten: Erstens ist der Stellenwert der IPÖ-Forschung innerhalb der politikwissenschaftlichen Forschung deutlich angestiegen; zweitens werden auch in öffentlichen Debatten politökonomische Fragestellungen verstärkt aufgegriffen.

Dieser Bedeutungsgewinn der IPÖ ist für uns Anlass, mit diesem Band eine Art Zwischenbilanz zu ziehen und der Frage nachzugehen, wo die IPÖ gegenwärtig steht und welche Forschungsperspektiven sich im Kontext der Weltfinanzkrise - vor allem für die deutsche IPÖ - eröffnen. 


\section{Themen und Forschungsfelder der IPÖ}

Im ersten Teil dieses Bandes sollen verschiedene theoretische Zugänge in Bezug zur Weltfinanzkrise gesetzt werden. Die Rückwirkungen der empirischen Ereignisse auf die Theorieentwicklung werden dabei unterschiedlich bewertet. Zum Teil gibt es durch die Krise einfach nur ein Mehr an Ereignissen, das es anhand der bewährten Ansätze zu analysieren gilt. Darüber hinaus werden aber auch grundlegende Annahmen erschüttert, was zu neuen Forschungsfragen und Theoriekonzeptionen führt.

Michael Franke analysiert die Veränderungen der Global Economic Governance (GEG) unter dem Blickwinkel des Societal Approach. Von der Analyse gesellschaftlicher Präferenzbildung auf nationaler Ebene ausgehend werden die Änderungen der GEG am Beispiel der Bankenregulierung erklärt. Die Positionen der nationalen Regierungen in internationalen Verhandlungen werden in diesem Ansatz durch gesellschaftliche Interessen und Ideen bestimmt. So resultiert die deutsche Haltung in den Basel III-Verhandlungen aus der nationalen Interessenkonstellation, die insbesondere durch die spezifische deutsche Bankenstruktur, aber auch durch die Bedürfnisse der Realwirtschaft und des Mittelstandes entsteht, während die britische Position hauptsächlich von den Interessen der City of London geprägt ist.

Aus einer radikal-konstruktivistischen Perspektive reflektiert Oliver Kessler die theoretischen Implikationen der Weltfinanzkrise. Gerade durch die Globalität der Finanzmärkte ist eine Analyse der sie konstituierenden Normen und Diskurse unabdingbar. Dazu beleuchtet der Autor den Unsicherheitsbegriff und dessen spezifische Konzeptualisierung. Während in der rationalistischen Forschung Unsicherheit mit Risiko gleichgesetzt wird, stützt sich eine eher moderate konstruktivistische Theorieschule auf keynesianische Überlegungen zu (Erwartungs-)Unsicherheit und Wahrscheinlichkeit. Diesen Fokus dekonstruiert Kessler, indem er die inneren Widersprüche zwischen Keynes' logischem Positivismus und dem konstruktivistischen wissenschaftlichen Anspruch aufzeigt. Hinzu kommt eine Trennung des Politischen vom Sozialen, die schwerwiegende gesellschaftliche Folgen haben kann. Kessler skizziert daher eine radikal-konstruktivistische Forschungsagenda, die sich vom Keynesianischen Unsicherheitsbegriff abwendet und das Politische und das Soziale zusammendenkt.

Auch Andreas Nölke und Christian May sehen angesichts der Weltfinanzkrise eine Neuorientierung der Forschung als erforderlich an. Im Bereich der von ihnen betrachteten vergleichenden Kapitalismusforschung sollte dies, so ihr Argument, darauf hinaus laufen, sich nicht allein auf den inter-nationalen Vergleich, sondern sehr viel stärker auf den intertemporalen Vergleich zu konzentrieren. Ihr „kritischer Institutionalismus“, der sich aus verschiedenen theoretischen Überlegungen - von Marx über Weber, Polanyi und Keynes bis hin zu jüngeren Beiträgen aus dem Umfeld der Weltsystem- und Regulationstheorie - speist, erkennt dabei ein Muster von sich abwechselnden kapitalistischen Phasen der Organisierung und Liberalisierung. Die Krise ist danach Ausdruck und Beginn eines weiteren Umschwungs, in dem das Pendel vom Liberalismus wieder zu einer organisierten Phase umschwenkt. Dabei sind vor allem die BRIC-Staaten für die neue Phase prägend. Die Autoren prognostizieren damit den Aufstieg eines organisierten Staatskapitalismus.

Stefan Schmalz zeigt in seinem Text die Alleinstellungsmerkmale einer historisch materialistischen IPÖ (hm IPÖ) im Kontext der Krise auf: die starke Fokussierung auf 
herrschaftssoziologische Aspekte und die geopolitischen Dimensionen der Krise. Was die Herrschaftssoziologie betrifft, so verdeutlicht die hm IPÖ, dass sich über eine Verschiebung der Kräfteverhältnisse und die Internationalisierung des Staates eine transnationale Kapitalismusformation herausgebildet hat, die zugunsten der VermögensbesitzerInnen und des Finanzkapitals ausfällt. Geopolitisch ist festzustellen, dass China und weitere Schwellenländer im Vergleich zu den USA immer mehr an Bedeutung gewinnen. Diese hegemoniale Transition findet aber innerhalb des transnationalen Kapitalismus statt und hat deshalb nicht zwangsläufig eine grundlegende Veränderung desselben zur Folge.

Neben den theoretischen Debatten stellen sich auch Fragen der räumlichen Auswirkungen der Weltfinanzkrise. Obwohl sich die Finanzkrise global ausbreitete, ist die regionale Betroffenheit unterschiedlich stark und unterschiedlich langanhaltend. Damit verbunden drängt sich außerdem die Frage nach möglichen geopolitischen Machtverschiebungen und regionalen Entwicklungen auf. Diese werden im zweiten Heftteil näher beleuchtet.

Christoph Scherrer setzt in seinem Beitrag zur Debatte um die erodierende Hegemonie der USA einen Konterpunkt. Er kommt aus einer gramscianischen Perspektive zu dem Schluss, dass die hegemoniale Stellung der USA zwar durch die Krise in einigen Bereichen etwas geschwächt wurde, im Großen und Ganzen ist ein Niedergang des Hegemons mit Blick auf die Bereiche Militär, Wirtschaft, Kultur und Institutionen aber nicht zu beobachten. Dies liegt vor allem daran, dass es sich um eine ,verschränkte Hegemonie‘ handelt, bei der die Interessen der USA mit den Interessen einer emergenten internationalen Bourgeoisie zusammenfallen, wodurch eine Einbindung der BRIC-Staaten erfolgt.

Auch für die Europäische Union (EU) sieht Hubert Zimmermann keinen umfassenden Bedeutungsverlust. Zu diesem Ergebnis kommt er über eine Analyse der Macht der EU in verschiedenen Politikfeldern. Dabei schreibt er dem jeweiligen Politikfeld eine spezifische Machtdimension zu: Konkret untersucht er erstens die Gemeinsame Sicherheits- und Verteidigungspolitik unter Verwendung eines klassischen Machtbegriffs, der vor allem militärische Ressourcen in den Blick nimmt; zweitens die Außenhandelspolitik der EU mithilfe eines strukturellen Machtbegriffs, der die Fähigkeit zur Rahmen- und Regelgebung hervorhebt; drittens die Währungspolitik mit Blick auf einen Machtbegriff, der Autonomie als zentrales Kennzeichen einer dominanten Stellung herausstellt und schließlich viertens die Umweltpolitik der EU mittels eines normativen Machtbegriffs, der vor allem auf den Vorbildcharakter und die Möglichkeit abzielt, moralische Normen zu verbreiten. Die Auswirkungen der Krise stellen sich für ihn bereichsspezifisch sehr unterschiedlich dar, weshalb nicht pauschal von einem Machtschwund der EU gesprochen werden kann.

China wiederum kommt im ostasiatischen Raum eine immer bedeutendere Rolle zu. Dirk Nabers untersucht in seinem Beitrag aus einer poststrukturalistisch inspirierten Perspektive den Nexus zwischen Außenwirtschaftspolitik und Identitätsbildung. Er zeigt auf, wie sich über die zunehmende wirtschaftliche Kooperation im Rahmen der ASEAN+3 der Begriff Ostasien zu einer gemeinsamen Identität verdichtet, die aber nicht notwendigerweise homogen ist. Wie im Anschluss an die Asienkrisen, so wird auch im Kontext der Weltfinanzkrise die Zusammenarbeit weiter verstärkt. Für Nabers wird letztlich ein Identitätswandel weg vom Prinzip der Nicht-Einmischung hin zur wechselseitigen wirtschaftspolitischen Anteilnahme im ostasiatischen Raum vollzogen. Die weitere Verdichtung dieses Wandels wird maßgeblich von China geprägt sein. 
Lateinamerika beweist in der Krise, so Joachim Becker, Johannes Jäger und Bernhard Leubolt in ihrem Beitrag, eine fragile Stabilität. Aufgrund der Krisenanfälligkeit finanzialisierter Entwicklungspfade haben zahlreiche Regierungen ihre Wirtschaftspolitik stärker auf die Industrialisierung und Binnenmarktorientierung ausgerichtet. Die MercosurLänder waren zumeist eher schwach von der Krise betroffen. Andere wiederum waren über ihre Abhängigkeit von der US-Wirtschaft (Mexiko und Mittelamerika) und/oder die Abhängigkeit von Rohstoffpreisen (Andenländer) stärker von der Krise betroffen. Durch die Krise haben sich die wirtschaftlichen Verflechtungen der Region mit China intensiviert. Generell ist aber auch hier abzuwarten, wie sich diese Beziehungen weiter entwickeln. Vorerst scheinen die aktuellen Entwicklungspfade vieler lateinamerikanischer Länder stabiler zu sein als noch vor 15 Jahren.

Die Tiefe und der Umfang der krisenbedingten Veränderungen werden von den Autoren recht unterschiedlich gesehen. Jenseits aller Differenzen sind sie sich aber einig, dass durch die Krise einige der bereits laufenden Transformationen eher verstärkt als abgeschwächt worden sind. In der globalen Konstellation ist aber nicht nur eine räumlich geprägte Forschung interessant. Vielmehr befasst sich die IPÖ auch mit politikfeldspezifischen Themen und Forschungsschwerpunkten, die eine strikte nationale Fokussierung aufbrechen und überwinden. Im dritten Teil werden daher exemplarisch einige globale und transnationale Dimensionen analysiert.

Brigitte Young analysiert in ihrem Beitrag die Ansätze der Reform der internationalen Finanzarchitektur im Rahmen der G20. Nachdem das Forum kurz nach dem Ausbruch der Krise über eine weitgehende Reregulierung der Finanzmärkte debattierte, konnten die FinanzmarktakteurInnen schnell ihren Einfluss auf die Agenda zurückgewinnen und viele Vorhaben abschwächen oder gar verhindern. Die G20 konnten zwar Veränderungen in Bezug auf die Befugnisse der Finanzaufsicht, Bankenregulation und auch Steuerhinterziehung anstoßen, deren Reichweite bleibt bislang aber eher begrenzt. So wurde die globale Finanzarchitektur trotz ihrer offenbar gewordenen Krisenanfälligkeit nicht grundlegend transformiert.

Entgegen mancher Befürchtungen hat die Weltfinanzkrise im Bereich der Handelspolitik nicht zu einem zunehmenden Protektionismus geführt und der Welthandel erholte sich nach einem kurzen Einbruch sehr schnell. Diesem Forschungspuzzle gehen Maria Behrens und Holger Janusch in ihrem Beitrag nach. Die AutorInnen erklären diese Entwicklung mit dem Konzept der Transnationalisierung des Staates. Die Reorganisation von Staatlichkeit im Kontext der Globalisierung geht einher mit einem wachsenden Einfluss transnationaler Unternehmen, die wiederum für einen Großteil des Welthandels verantwortlich sind. Diese Unternehmen haben kein Interesse an protektionistischen Maßnahmen und konnten diese weitgehend verhindern.

In ihrem Beitrag beleuchten Marian Feist und Doris Fuchs die Auswirkungen der Finanzkrise auf die Agrarpolitik und Ernährungssicherheit. Da durch die Finanzkrise vorerst viele Renditemöglichkeiten wegfielen, suchten sich FinanzmarktakteurInnen neue Anlagenfelder, unter anderem die Spekulation mit Nahrungsmitteln. Diese verursachten eine starke Preissteigerung, die in vielen Entwicklungsländern zu Hungerkrisen führte. Auch die Praxis des Land-Grabbing stellt eine Entwicklung dar, die verheerende Folgen für die ländliche Bevölkerung mit sich bringt. Versuche, diese Problematiken über die Regulation der Agrarmärkte abzuschwächen, blieben bisher erfolglos. Dies ist mit asym- 
metrischen Machtverhältnissen zu erklären, in denen all jene, die kein Regulierungsinteresse haben, über eine umfassend entwickelte instrumentelle, strukturelle und materielle Macht verfügen. Zugleich ist durch die Finanzkrise aber der Handlungsbedarf gewachsen, globale Ernährungssicherheit zu gewährleisten und so bleiben mögliche Entwicklungen abzuwarten.

Auch im Bereich der Klima- und Energiepolitik ist ein Wechselspiel mit der Finanzund Wirtschaftskrise zu beobachten. Achim Brunnengräber und Tobias Haas analysieren in ihrem Beitrag am Beispiel der EU die Kohärenzprobleme von klima- und energiepolitischen Maßnahmen und verweisen auf die Hindernisse, die einer great transformation bzw. einer green economy entgegenstehen. Aufgrund der spezifischen Kräfteverhältnisse, die von grünen (erneuerbare Energien) und grauen (fossilistische und atomare Energien) Akteurskoalitionen strukturiert werden, geht der Wandel hin zu einem Regime erneuerbarer Energien nur sehr langsam vonstatten. Die grauen Akteurskoalitionen sind nach wie vor in der Lage, die europäische Agenda maßgeblich zu beeinflussen. Die Eurokrise kommt in zahlreichen Ländern erschwerend hinzu, da die Förderung erneuerbarer Energien im Zuge der Austeritätspolitik vielfach stark reduziert wird.

Die Krisenprozesse und die spezifische Krisenbearbeitung haben allerdings nicht nur politikfeldspezifische sondern auch gesellschaftspolitische Auswirkungen. Abschließend beleuchten Hans-Jürgen Bieling, Tobias Haas und Julia Lux die Widerstandsprozesse, die sich im Euroraum entwickelt haben. Dabei stellen sie räumlich und zeitlich unterschiedliche Protestkonjunkturen fest. Während die wirtschaftlichen und sozialen Folgen der Krise und die negativen sozialen, demokratischen, ökologischen und integrationsspezifischen Folgen des europäischen Krisenmanagements in den stärker von der Krise betroffenen südeuropäischen Defizitländern der Eurozone zu starken Protesten führen, entwickelt sich in den nordeuropäischen Überschussländern nur eine zaghafte Kritik an der Austeritätspolitik. Es gelingt weder den Gewerkschaften noch den sozialen Bewegungen, öffentlich kraftvoll für einen alternativen gesellschaftlichen Entwicklungspfad zu mobilisieren. Bisher ist daher nur ein fragmentierter europäischer Konfliktzyklus zu beobachten.

Insgesamt stellt sich das Zusammenspiel zwischen der Finanz- und Wirtschaftskrise und verschiedenen Politikbereichen sehr unterschiedlich dar. In einigen Bereichen verschärft die Krise bestehende Probleme und ruft eine Krisenbearbeitung hervor, die je nach Deutung der Ursachen der Krise sehr unterschiedlich ausfällt und deren Wirkungen ein weiteres Forschungsfeld für die IPÖ bedeuten. Der Fortgang der politikfeldspezifischen, aber auch der räumlichen und geopolitischen Entwicklungen bleibt daher zu beobachten.

Danksagungen: Besonderer Dank gilt an dieser Stelle Jannis Chasoglou und Corinna Hillebrand-Brem sowie Milan Babić, die uns bei der redaktionellen Bearbeitung dieses Heftes tatkräftig unterstützt haben.

\section{Literatur}

Becker, J., \& Jäger, J. (2012). Integration in crisis: A regulationist perspective on the interaction of European varieties of capitalism. Competition \& Change, 16(3), 169-187. 
Bellofiore, R., Garibaldo, F., \& Halevi, J. (2010). The global crisis and the crisis of European neomercantilism. In L. Panitch, A. Gregory \& V. Chibber (Hrsg.), The crisis this time. Socialist register 2011. (S. 120-146). London: Merlin Press, Monthly Review Press, Fernwood Publishing.

Bieling, H-J. (2009). Wenn der Schneeball ins Rollen kommt: Überlegungen zur Dynamik und zum Charakter der Subprime-Krise. Zeitschrift für Internationale Beziehungen, 16(1), 107-121.

Bieling, H-J. (2011). Internationale Politische Ökonomie. Eine Einführung, 2. aktualisierte Aufl. Wiesbaden: VS Springer.

Biersteker, T. J. (1993). Evolving perspectives on International political economy: Twentieth-century contexts and discontinuities. International Political Science Review, 14(1), 7-33.

Cohen, B. J. (2007). The transatlantic divide: Why are American and British IPE so different? Review of International Political Economy, 14(2), 197-219.

Cohen, B. J. (2008). International political economy. An intellectual history. Princeton: Princeton University Press.

Gilpin, R. (1987). The political economy of International relations. Princeton: Princeton University Press.

Kosellek, R. (1973). Kritik und Krise. Frankfurt a. M.: Suhrkamp.

Phillips, N., \& Weaver, C. E. (Hrsg.). (2011). International political economy. Debating the past, present, and future. New York: Routledge.

Schirm, S. (2013). Internationale Politische Ökonomie. Eine Einführung, 3. überarbeitete und erweiterte Aufl. Baden-Baden: Nomos. 